\title{
Transmission of Severe Acute Respiratory Syndrome Coronavirus 2 to Close Contacts, China, January-February 2020
}

Yu Li, ${ }^{1}$ Jianhua Liu, ${ }^{1}$ Zhongcheng Yang, ${ }^{1}$ Jianxing Yu, Chengzhong Xu, Aiqin Zhu, Hao Zhang, Xiaokun Yang, Xin Zhao, Minrui Ren, Zhili Li, Jinzhao Cui, Hongting Zhao,

Xiang Ren, Chengxi Sun, Ying Cheng, Qiulan Chen, Zhaorui Chang, Junling Sun, Lance E. Rodewald, Liping Wang, Luzhao Feng, George F. Gao, ${ }^{2}$ Zijian Feng, ${ }^{2}$ Zhongjie Li

We estimated the symptomatic, PCR-confirmed secondary attack rate (SAR) for 2,382 close contacts of 476 symptomatic persons with coronavirus disease in Yichang, Hubei Province, China, identified during January 23-February 25, 2020. The SAR among all close contacts was 6.5\%; among close contacts who lived with an index case-patient, the SAR was $10.8 \%$; among close-contact spouses of index case-patients, the SAR was $15.9 \%$. The SAR varied by close contact age, from $3.0 \%$ for those $<18$ years of age to $12.5 \%$ for those $\geq 60$ years of age. Multilevel logistic regression showed that factors significantly associated with increased SAR were living together, being a spouse, and being $\geq 60$ years of age. Multilevel regression did not support SAR differing significantly by whether the most recent contact occurred before or after the index case-patient's onset of illness ( $p$ $=0.66$ ). The relatively high SAR for coronavirus disease suggests relatively high virus transmissibility.

$\mathrm{T}$ ransmissibility of an emerging infectious disease is a key factor for determining transmission dynamics in a population. The basic reproductive number, $\mathrm{R}_{0^{\prime}}$ indicates the average number of new cases resulting from 1 infected person in a completely susceptible population (1). In December 2019, an outbreak of coronavirus disease (COVID-19), caused by severe acute respiratory syndrome coronavirus

Author affiliations: Chinese Center for Disease Control and Prevention, Beijing, China (Y. Li, J. Yu, A. Zhu, X. Yang, M. Ren, Zhili Li, J. Cui, H. Zhao, X. Ren, C. Sun, Y. Cheng, Q. Chen, Z. Chang, J. Sun, L.E. Rodewald, L. Wang, L. Feng, G.F. Gao, Z. Feng, Zhongjie Li); Yichang Center for Disease Control and Prevention, Yichang, China (J. Liu, Z. Yang, C. Xu, H. Zhang, X. Zhou)

DOI: https://doi.org/10.3201/eid2709.202035
2 (SARS-CoV-2), was identified in Wuhan, Hubei Province, China (2). The mean $\mathrm{R}_{0}$ of COVID-19 was estimated to be in the range of 1.90-6.49 (3), indicating a high contagiousness that led to its rapid spread across the world (4). Another indicator of infectiousness is secondary attack rate (SAR), which is the probability that infection occurs among susceptible persons within a reasonable incubation period after known contact with an infectious person or an infectious source $(5,6)$. Few estimates are available for the SAR for COVID-19 and its variation by type of contact, characteristics of index case-patients and contacts, and other factors. Information about factors associated with variation in SAR could help identify persons at high risk of transmitting the virus or acquiring COVID-19. Studies have reported transmission during the incubation period of COVID-19 (7-10) but with unclear quantification of risk. We estimated the SAR for COVID-19 and factors associated with risk for transmission.

\section{Methods}

We conducted this study from January 23 through February 25, 2020, in Yichang, Hubei Province, China; the city has a population of $\approx 4$ million. In accordance with National Health Commission guidelines for prevention and control of COVID-19 (http://www. gov.cn/xinwen/2020-01/23/content_5471768.htm), close contacts of COVID-19 case-patients were placed under 14-day quarantine for medical observation, during which time they would be tested by PCR for SARS-CoV-2 one time if illness symptoms developed

\footnotetext{
${ }^{1}$ These authors contributed equally to this article.

${ }^{2}$ These authors are the senior authors.
} 
but not tested if illness symptoms did not develop during the quarantine period.

Nasopharyngeal and pharyngeal swab samples from symptomatic quarantined persons were obtained and placed in airtight, freeze-tolerant tubes containing $3.5 \mathrm{~mL}$ of UTM (universal transport medium) viral transport medium. Sealed tubes were transported to the Yichang Center for Disease Control and Prevention laboratory (Yichang, China) within 24 hours of specimen collection. Viral RNA was extracted from samples and tested by using a commercial SARS-CoV-2 PCR diagnostic kit (Bioperfectus Technologies, https://www.bioperfectus.com) according to the manufacturer's instructions. The commercial kit targets the open reading frame $1 \mathrm{ab}$ and nucleocapsid protein genes of the SARS-CoV-2 genome.

An index case-patient was defined as a person in this study with a positive SARS-CoV-2 PCR result. A close contact was defined as someone who had contact with an index case-patient without effective protection and within 1 meter, regardless of contact duration. Persons who had close contact with the index case-patient during or 2 days before the index casepatient's illness onset were counted as close contacts. Secondary case-patients were close contacts with positive SARS-CoV-2 test results.

The types of contacts were considered mutually exclusive and were living together in the same household as an index case-patient, eating together (having meals together at a party, in a restaurant, or in another setting), caring for a patient (including doctors, nurses, and family members taking care of patients), sharing a vehicle (riding the same vehicle with an index case-patient but with no other close contact), or staying in a confined space (in the same confined space with an index case-patient, excluding in a vehicle, and with no other close contact). We included in our analyses close contacts who had completed their 14-day quarantine or who had positive SARS$\mathrm{CoV}-2$ results during quarantine in our study period. We excluded from our analyses close contacts of suspected case-patients for whom laboratory evidence of COVID-19 was lacking. We also excluded close contacts of $>1$ index case-patient or those whose information about contact type was missing.

We estimated the SAR by dividing the number of secondary cases by the number of close contacts. SAR in our study refers to secondary case-patients who had symptomatic, PCR-confirmed infection. We estimated the SAR for each type of close contact, tested statistical significance of differences by using $\chi^{2}$ or Fisher exact tests as appropriate, and considered $\mathrm{p}<0.05$ to be significant. We further analyzed factors significantly associated with SAR in univariate analyses with multilevel logistic regression mixed-effect models. We estimated crude and adjusted odds ratios (ORs) and 95\% CIs, accounting for random effects of index case-patients.

Surveillance and analysis of close contacts of COVID-19 case-patients is part of public health surveillance in China. These procedures are exempted from need for institutional review board approval.

\section{Results}

We included in our analyses 2,382 close contacts of 476 symptomatic index case-patients, all of whom completed their 14-day quarantine with assessed outcomes and who provided contact-related information (Figure). Close contacts were generally younger and more likely to be female than their corresponding

\section{2,762 contacts of 533 index case-patients}

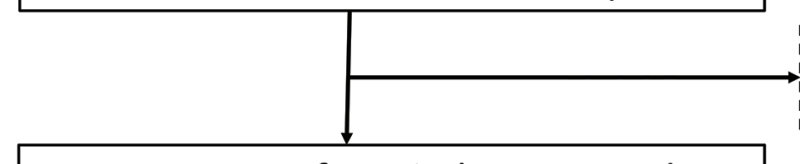

2,606 contacts of 533 index case-patients

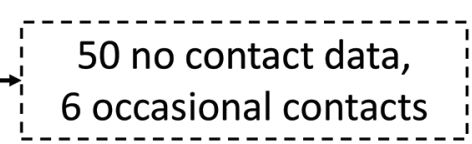

-

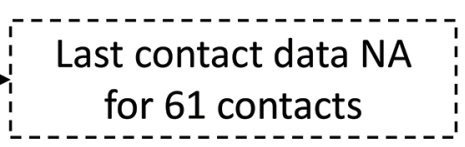

163 contacts with multiple index case-patients
Figure. Enrollment of close contacts in study of transmission

of severe acute respiratory syndrome coronavirus 2 to close contacts, China, JanuaryFebruary 2020.

2,545 contacts of 495 index case-patients

2,382 contacts of 476 index case-patients 
Table 1. Characteristics of 476 index case-patients and 2,382 close contacts in study of transmission of severe acute respiratory syndrome coronavirus 2 to close contacts, China, January-February $2020^{*}$

\begin{tabular}{lcc}
\hline Characteristic & $\begin{array}{c}\text { Index case- } \\
\text { patients }\end{array}$ & Close contacts \\
\hline Age, y, mean (range) & $49(2-91)$ & $43(0-94)$ \\
\hline $\begin{array}{l}\text { Age group, y } \\
\quad<18\end{array}$ & $5(1)$ & $267(12)$ \\
$18-59$ & $339(71)$ & $1,559(68)$ \\
$\geq 60$ & $132(28)$ & $465(20)$ \\
\hline Sex & $262(55)$ & $1,162(49)$ \\
$\quad$ M & $214(45)$ & $1,198(51)$ \\
$\quad \mathrm{F}$ & & \\
\hline${ }^{*}$ Values are no. (\%) unless otherwise indicated. & \\
\hline
\end{tabular}

index case-patients (Table 1). The overall SAR was $6.5 \%$. SAR was $10.8 \%$ among close contacts who lived together with an index case-patient; this rate was significantly higher than that for other contact types, for which SAR ranged from $1.5 \%$ to $4.0 \%$ (Table 2). The SAR was $15.9 \%$ among spouses of index case-patients. SAR did not differ by sex of close contacts or of index case-patients. SAR increased with age, from 3.0\% among close contacts $<18$ years of age to $12.5 \%$ among close contacts $\geq 60$ years of age. A similar pattern by age was found for index case-patients (Table 2).

The SAR was $4.7 \%$ for close contacts whose most recent contact with an index case-patient was during the index case-patient's incubation period, compared with a SAR of $7.3 \%$ for close contacts for whom the most recent contact occurred after index case-patient illness onset $(p=0.023)$. In multilevel univariate analysis that accounted for index case-patient variation, the pattern of ORs for factors associated with SAR was similar to the pattern described above (Table 3). In multilevel analysis that used a multivariate model with age of close contact, adjusted ORs for the following differed slightly from those for the univariate analysis: age of index case-patient, type of contact, whether the close contact and the index case-patient were spouses, and most recent contact time between close contact and index case-patient. Associations between SAR and the most recent contact time with the index case-patient (before/after illness onset) and age of the index case-patients ( $<60$ years $>60$ years) were no longer statistically significant, although the directions of the associations were the same (Table 3). The associations of SAR with age of contact, living together with an index case-patient, and being the spouse of an index case-patient were still significant, although the point estimates of the adjusted ORs became smaller (Table 3).

\section{Discussion}

We found the SAR among all close contacts to be $6.5 \%$. Because confirmed case-patients were centrally

\begin{tabular}{|c|c|c|c|}
\hline 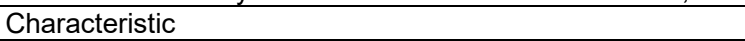 & Close contacts, no. & Secondary cases, no. (\%) & p value \\
\hline Overall & 2,382 & $156(6.5)$ & \\
\hline \multicolumn{4}{|l|}{ Contact } \\
\hline Type of contact & & & $<0.001$ \\
\hline Living together & 1,020 & $110(10.8)$ & \\
\hline Eating together & 835 & $33(4.0)$ & \\
\hline Care & 80 & $2(2.5)$ & \\
\hline Sharing vehicle & 68 & $1(1.5)$ & \\
\hline Stay in a confined space & 379 & $10(2.6)$ & \\
\hline Most recent contact with index case-patient & & & 0.023 \\
\hline Before illness onset & 686 & $32(4.7)$ & \\
\hline After illness onset & 1,696 & $124(7.3)$ & \\
\hline Whether contacts and index case-patients were spouses & & & $<0.001$ \\
\hline No & 2,105 & $112(5.3)$ & \\
\hline Yes & 277 & $44(15.9)$ & \\
\hline \multicolumn{4}{|l|}{ Close contacts } \\
\hline Age & & & $<0.001$ \\
\hline$<18 y$ & 267 & $8(3.0)$ & \\
\hline $18-59$ y & 1,559 & $89(5.7)$ & \\
\hline$\geq 60 y$ & 465 & $58(12.5)$ & \\
\hline $\operatorname{Sex}$ & & & 0.644 \\
\hline M & 1,162 & $71(6.1)$ & \\
\hline $\mathrm{F}$ & 1,198 & $83(6.9)$ & \\
\hline \multicolumn{4}{|l|}{ Index case-patients } \\
\hline Age & & & $<0.001$ \\
\hline$<18 \mathrm{y}$ & 86 & 0 & \\
\hline $18-59 y$ & 1,747 & $90(5.2)$ & \\
\hline$\geq 60 y$ & 549 & 66 (12.0) & \\
\hline Sex & & & 0.704 \\
\hline M & 1,303 & $82(6.3)$ & \\
\hline $\mathrm{F}$ & 1,079 & $74(6.9)$ & \\
\hline
\end{tabular}


Table 3. Univariate and multivariate analyses of factors associated with secondary attack rate for coronavirus disease, China, January-February 2020*

\begin{tabular}{|c|c|c|c|c|}
\hline \multirow[b]{2}{*}{ Characteristic of contact } & \multicolumn{2}{|c|}{ Univariate } & \multicolumn{2}{|c|}{ Multivariate† } \\
\hline & Crude OR $(95 \% \mathrm{Cl})$ & $p$ value & Adjusted OR $(95 \% \mathrm{Cl})$ & $\mathrm{p}$ value \\
\hline \multicolumn{5}{|l|}{ Type of contact } \\
\hline Not living together & Referent & & Referent & \\
\hline Living together & $7.85(3.89-15.83)$ & $<0.01$ & $5.12(2.11-12.45)$ & $<0.01$ \\
\hline \multicolumn{5}{|c|}{ Spouse of index case-patient } \\
\hline No & Referent & & Referent & \\
\hline Yes & $6.46(3.30-12.61)$ & $<0.01$ & $2.83(1.31-6.11)$ & $<0.01$ \\
\hline \multicolumn{5}{|l|}{ Age of contact, $y$} \\
\hline$<60$ & Referent & & Referent & \\
\hline$\geq 60$ & $3.29(1.86-5.82)$ & $<0.01$ & $2.61(1.43-4.78)$ & 0.01 \\
\hline \multicolumn{5}{|c|}{ Age of index case-patient, $y$} \\
\hline$<60$ & Referent & & Referent & \\
\hline$\geq 60$ & $5.13(1.66-15.86)$ & $<0.01$ & $2.92(0.80-10.59)$ & 0.1 \\
\hline \multicolumn{5}{|c|}{ Most recent contact with index case-patient } \\
\hline Before illness & Referent & & Referent & \\
\hline After illness & $2.20(1.06-4.59)$ & 0.03 & $1.23(0.49-3.07)$ & 0.66 \\
\hline
\end{tabular}

isolated and away from home, the SAR we measured may be lower than it would have been under conditions of home isolation. Factors independently associated with significantly higher risk of contracting COVID-19 were living in the same house as an index case-patient, being a spouse of an index case-patient, and being older. We found evidence of presymptomatic transmission, in which close contacts who only had contact with a COVID-19 case-patient during the incubation period subsequently had positive SARSCoV-2 test results. The SAR among these close contacts was $4.7 \%$, significantly lower than that for contacts whose most recent contact occurred after illness onset of the index case-patient.

We estimated the COVID-19 SAR in a household to be $10.8 \%$, slightly higher than SAR estimates for seasonal influenza and pandemic influenza (H1N1) viruses in Hong Kong (11). Our results suggest that transmissibility of SARS-CoV-2 might be similar or slightly higher than that of influenza virus, which has a SAR of $\approx 10 \%$ in the household setting (11). This similarity is consistent with the finding that the $\mathrm{R}_{0}$ for COVID-19 is also similar to or slightly higher than that for influenza (12). In contrast, the SAR in households is estimated to be $2 \%-7 \%$ for Middle East respiratory syndrome (13) and $6.2 \%$ for severe acute respiratory syndrome (14), suggesting slightly weaker transmissibility compared with COVID-19.

Our findings corroborated transmissibility of SARS-CoV-2 during the COVID-19 incubation period. Viral shedding has been observed during the COVID-19 incubation period $(15,16)$. Our results were consistent with those of another study that estimated that $40 \%$ of the transmission events in COVID-19 clusters were attributed to presymptomatic virus transmission in China (17). Our multivariate analysis did not find statistically significant differences in SAR before and after illness onset, which is consistent with a SAR study in southern China that found infectivity during the incubation period to not differ statistically from infectivity after illness onset. Although respiratory signs such as coughing and sneezing after illness onset increased the probability of virus transmission compared with during the incubation period (18-20), studies suggest that viral load peaks right before illness onset $(10,21)$, highlighting the threat for presymptomatic SARS-CoV-2 transmission.

Risk of contracting COVID-19 was positively associated with intimacy between contacts and index case-patients. Living in the same household with index case-patients considerably increased risk for COVID-19. Being a spouse of an index case-patient independently increased the risk of contracting COVID-19, consistent with findings from another study ( $P$. Cui et al., unpub. data, https:/ / www.medrxiv.org/ content/10.1101/2020.02.26.20028225v2). However, the SAR was relatively low among contacts who provided care to patients, implying that risk for infection can be reduced by using protective equipment and by protective behaviors.

Previous studies indicated that age was associated with risk for severe and fatal infection (22); however, few studies directly assessed the effect of age on risk of contracting COVID-19. Our study confirmed that senior persons are at high risk for contracting COVID-19, highlighting the need to pay special attention to facilities with numerous seniors, such as nursing homes. However, our findings also suggested that older age does not necessarily increase the risk of transmitting the virus; our multivariate analysis 
found that the association between older age of index case-patients and SAR was not statistically significant, a finding consistent with a study showing that viral loads did not differ significantly by age (10).

The first limitation of our study is that for surveillance of close contacts, laboratory testing was initiated only when the contacts showed symptoms of illness. Asymptomatic infections with SARS-CoV-2 occur; for example, 1 study estimated that $17.9 \%$ of persons infected with SARS-CoV-2 did not have any symptoms (23). Therefore, our study will have missed asymptomatic case-patients and therefore underestimated the true SAR. Our estimates should therefore be interpreted as SAR limited to secondary case-patients with symptomatic COVID-19. The second limitation is that SAR is determined not only by infectiousness of the virus but also by protection levels, which might differ by geography, phase of the pandemic, education level of persons at risk, perceived threat from COVID-19, and other confounding factors. The third limitation is that the number of index case-patients $<18$ years of age and corresponding contacts was small; thus, our estimates of SAR for COVID-19 are more representative of transmissibility among adults than among children.

In conclusion, the SAR for COVID-19 is relatively high, suggesting relatively high transmissibility. This SAR is influenced by type of contact, level of intimacy between case-patients and contact, and age of contact. Our results provide additional evidence that SARS-CoV-2 can be transmitted by presymptomatic persons.

\section{Acknowledgments}

We thank the Yichang Center for Disease Control and Prevention (CDC) for assistance with data collection. The views expressed are those of the authors and do not necessarily represent the policy of the China CDC.

This study was supported by the Emergency Response Mechanism Operation Program, China CDC (no. 131031001000015001). The funding bodies had no role in study design, data collection and analysis, preparation of the manuscript, or the decision to publish.

Zhongjie Li, Z.F., J.L., and G.F.G. conceived, designed, and supervised the study. Y.L. J.L., J.Y., and Zhongjie Li designed the study, finalized the analysis, and interpreted the findings. Y.L., J.L., Z.Y., A.Z., X.Y., Zhili Li, and J.C. wrote the initial drafts of the manuscript. M.R., C.X., H. Zhang., Z.X., and H. Zhao participated in collection and management of data. X.R., L.E.R., Y.C., Q.C., Z.C., J.S., L.W., C.S., and L.F. commented on and revised the final manuscript. All authors read and approved the submitted version of the manuscript.

\section{About the Author}

Dr. Yu Li is an epidemiologist in the Division of Infectious Diseases of China CDC. His primary interests include the epidemiology and transmission dynamics of hand, foot, and mouth disease; zoonotic and vector-borne diseases such as rabies, dengue, anthrax; and fever with thrombocytopenia syndrome.

\section{References}

1. Delamater PL, Street EJ, Leslie TF, Yang YT, Jacobsen $\mathrm{KH}$. Complexity of the basic reproduction number $\left(\mathrm{R}_{0}\right)$. Emerg Infect Dis. 2019;25:1-4. https://doi.org/10.3201/ eid2501.171901

2. Zhu N, Zhang D, Wang W, Li X, Yang B, Song J, et al.; China Novel Coronavirus Investigating and Research Team. A novel coronavirus from patients with pneumonia in China, 2019. N Engl J Med. 2020;382:727-33. https:/ / doi.org/ 10.1056/NEJMoa2001017

3. Alimohamadi $Y$, Taghdir M, Sepandi M. Estimate of the basic reproduction number for COVID-19: a systematic review and meta-analysis. J Prev Med Public Health. 2020;53:151-7. https://doi.org/10.3961/jpmph.20.076

4. Sanche S, Lin YT, Xu C, Romero-Severson E, Hengartner N, Ke R. High contagiousness and rapid spread of severe acute respiratory syndrome coronavirus 2. Emerg Infect Dis. 2020;26:1470-7. https://doi.org/10.3201/eid2607.200282

5. Yom-Tov E, Johansson-Cox I, Lampos V, Hayward AC. Estimating the secondary attack rate and serial interval of influenza-like illnesses using social media. Influenza Other Respir Viruses. 2015;9:191-9. https://doi.org/10.1111/ irv.12321

6. Yang Y, Sugimoto JD, Halloran ME, Basta NE, Chao DL, Matrajt L, et al. The transmissibility and control of pandemic influenza A (H1N1) virus. Science. 2009;326:729-33. https:// doi.org/10.1126/science.1177373

7. Buitrago-Garcia D, Egli-Gany D, Counotte MJ, Hossmann S, Imeri $\mathrm{H}$, Ipekci $\mathrm{AM}$, et al. Occurrence and transmission potential of asymptomatic and presymptomatic SARS-CoV-2 infections: a living systematic review and meta-analysis. PLoS Med. 2020;17:e1003346. https:// doi.org/10.1371/ journal.pmed.1003346

8. Furukawa NW, Brooks JT, Sobel J. Evidence supporting transmission of severe acute respiratory syndrome coronavirus 2 while presymptomatic or asymptomatic. Emerg Infect Dis. 2020;26. https://doi.org/10.3201/ eid2607.201595

9. Sun K, Wang W, Gao L, Wang Y, Luo K, Ren L, et al. Transmission heterogeneities, kinetics, and controllability of SARS-CoV-2. Science. 2021;371:24. https://doi.org/10.1126/ science.abe2424

10. He X, Lau EHY, Wu P, Deng X, Wang J, Hao X, et al. Temporal dynamics in viral shedding and transmissibility of COVID-19. Nat Med. 2020; 26:672-5. https:/ / doi.org/ 10.1038/s41591-020-0869-5

11. Cowling BJ, Chan KH, Fang VJ, Lau LLH, So HC, Fung ROP, et al. Comparative epidemiology of pandemic and seasonal influenza A in households. N Engl J Med. 2010;362:2175-84. https://doi.org/10.1056/NEJMoa0911530

12. White LF, Wallinga J, Finelli L, Reed C, Riley S, Lipsitch M, et al. Estimation of the reproductive number and the serial interval in early phase of the 2009 influenza A/H1N1 pandemic in the USA. Influenza Other Respir Viruses. 2009; 3:267-76. https://doi.org/10.1111/j.1750-2659.2009.00106.x 
13. Hui DS, Azhar EI, Kim YJ, Memish ZA, Oh MD, Zumla A. Middle East respiratory syndrome coronavirus: risk factors and determinants of primary, household, and nosocomial transmission. Lancet Infect Dis. 2018;18:e217-27. https://doi.org/10.1016/S1473-3099(18)30127-0

14. Goh DL, Lee BW, Chia KS, Heng BH, Chen M, Ma S, et al. Secondary household transmission of SARS, Singapore. Emerg Infect Dis. 2004;10:232-4. https:/ / doi.org/10.3201/ eid1002.030676

15. Hu Z, Song C, Xu C, Jin G, Chen Y, Xu X, et al. Clinical characteristics of 24 asymptomatic infections with COVID-19 screened among close contacts in Nanjing, China. Sci China Life Sci. 2020;63:706-11.

16. Pan $Y$, Zhang D, Yang P, Poon LLM, Wang Q. Viral load of SARS-CoV-2 in clinical samples. Lancet Infect Dis. 2020;20:411-2. https:/ / doi.org/10.1016/S1473-3099 (20)30113-4

17. Ren X, Li Y, Yang X, Li Z, Cui J, Zhu A, et al. Evidence for pre-symptomatic transmission of coronavirus disease 2019 (COVID-19) in China. Influenza Other Respir Viruses. 2020;15:19-26. https://doi.org/10.1111/irv.12787

18. Lindsley WG, Blachere FM, Thewlis RE, Vishnu A, Davis KA, Cao G, et al. Measurements of airborne influenza virus in aerosol particles from human coughs. PLoS One. 2010;5:e15100. https:/ / doi.org/10.1371/ journal.pone.0015100

19. To KK, Tsang OT, Yip CC, Chan KH, Wu TC, Chan JM, et al. Consistent detection of 2019 novel coronavirus in saliva. Clin Infect Dis. 2020;71:841-3. https:/ / doi.org/10.1093/ cid/ciaa149

20. Yan J, Grantham M, Pantelic J, Bueno de Mesquita PJ, Albert B, Liu F, et al.; EMIT Consortium. Infectious virus in exhaled breath of symptomatic seasonal influenza cases from a college community. Proc Natl Acad Sci U S A. 2018;115:1081-6. https:// doi.org/10.1073/pnas.1716561115

21. Arons MM, Hatfield KM, Reddy SC, Kimball A, James A, Jacobs JR, et al.; Public Health-Seattle and King County and CDC COVID-19 Investigation Team. Presymptomatic SARS-CoV-2 infections and transmission in a skilled nursing facility. N Engl J Med. 2020;382:2081-90. https://doi.org/10.1056/NEJMoa2008457

22. Zhou F, Yu T, Du R, Fan G, Liu Y, Liu Z, et al. Clinical course and risk factors for mortality of adult inpatients with COVID-19 in Wuhan, China: a retrospective cohort study. Lancet. 2020;395:1054-62. https://doi.org/10.1016/ S0140-6736(20)30566-3

23. Mizumoto K, Kagaya K, Zarebski A, Chowell G. Estimating the asymptomatic proportion of coronavirus disease 2019 (COVID-19) cases on board the Diamond Princess cruise ship, Yokohama, Japan, 2020. Euro Surveill. 2020;25. https:// doi.org/10.2807/1560-7917.ES.2020.25.10.2000180

Address for correspondence: Zhongiie Li, Chinese Center for Disease Control and Prevention, 155 Changbai Rd, Changping District, Beijing 102206, China; email: lizj@chinacdc.cn

\section{EID Podcast: Laboratory-Associated Zika Virus, United States}

Since the 2015 Zika virus outbreak in the Americas, transmission of this vectorborne disease has substantially decreased. But Zika virus doesn't spread only through mosquito bites...it also spreads through sexual transmission, blood transfusions, breastfeeding, and even needlestick injuries in laboratories.

Stringent safety protocols minimize the risk of laboratoryassociated exposures. But on rare occasions, researchers are accidentally exposed to the disease they are trying to solve.

In this EID podcast, Dr. Susan Hills, a medical epidemiologist at CDC in Fort Collins, Colorado, describes the biosafety lessons exemplified by four cases of laboratory-associated Zika infection. 